\title{
Enquête sur les Nématodes des Poissons de la région nantaise
}

\section{Identification des larves d'Ascarides parasitant les Sardines (en rapport avec les granulomes éosinophiles observés chez l'homme dans la région)}

\author{
par Annie J. PETTER \\ [Laboratoire de Zoologie (Vers) associé au C.N.R.S., Muséum National \\ d'Histoire Naturelle ( $\mathrm{P}^{\mathrm{r}}$ A.-G. Chabaud), 57, rue Cuvier, Paris $\left.\left(5^{\circ}\right)\right]$ \\ [Laboratoire de Parasitologie et Pathologie exotique ( $\mathrm{P}^{\mathrm{r}} \mathrm{C}$. VERMEIL) et \\ Laboratoire d'Anatomie Pathologique ( $\mathrm{P}^{\mathrm{r}}$ J.-P. Kerneis), Faculté de Médecine de Nantes, \\ 1, rue Gaston-Veil, 44 - Nantes]
}

\begin{abstract}
Résumé
Nous donnons les résultats d'une enquête sur les Nématodes récoltés chez des Poissons pêchés en juillet et août au Croisic (Bretagne); six espèces de Nématodes adultes et deux espèces de Nématodes larvaires ont été trouvés; nous donnons la description d'une espèce nouvelle de Cystidicolinae, Spinitectoides berlandi, parasite de Molva molva (L.); cette espèce, connue uniquement par les femelles, appartient à un nouveau genre, Spinitectoides, caractérisé par une ornementation cuticulaire en festons sur la région antérieure du corps et par une structure apicale voisine de celle de Cystidicola.

Nous décrivons l'aspect des larves à l'éclosion chez Thynnascaris aduncum et Thynnascaris cornutum; nous rappelons les caractères distinctifs entre les trois espèces de Thynnascaris trouvées: $T$. aduncum, $T$. rigidum et $T$. cornutum; les longueurs relatives de l'appendice œsophagien et du caecum intestinal, ainsi que l'aspect de l'appendice œsophagien en section transversale, sont semblables chez $T$. aduncum et chez les larves de Thynnascaris trouvées chez les Sardines [Sardina pilchardus (Walbaum)]; nous identifions donc les larves qui sont vraisemblablement à l'origine des granulomes éosinophiles humains comme larves de Thynnascaris aduncum.
\end{abstract}




\section{Summary}

The results of a survey of the Nematodes parasiting marine fishes collected in July and August off Le Croisic (Bretagne) are given.

Six species of adult Nematodes and two species of nematode larvae were obtained. A new species of Cystidicolinae parasite of Molva molva (L.) is described: Spinitectoides berlandi is known only by the females, it belongs to a new genus Spinitectoides which main characteristics are a festooned cuticular ornament on the anterior part of body and an apical structure closely similar to this of Cystidicola.

We describe the morphology of hatching larvae of Thynnascaris aduncum and Th. cornutum, and we record the main distinctive characters of three species of Thynnascaris; Th. aduncum, $T h$. rigidum and Th. cornutum. The relative length of both the oesophageal appendix and the intestinal caecum as well as the morphology of the oesophageal appendix viewed in transversal section are similar in Th. aduncum and in the Thynnascaris larvae collected from sardines [Sardina pilchardus (Walbaum)] : therefore we have identified as larvae of $T h$. aduncum the larvae which are presumably causing the eosinophilic granuloma of man.

Dans une note précédente (1969), nous exposions les résultats d'une enquête sur les Nématodes des Sardines pêchées dans la région nantaise, enquête effectuée en rapport avec l'existence de cas de granulomes éosinophiles observés chez l'homme dans la région (voir Picard et coll., 1954, Boquien et coll., 1966, Benatre et coll., 1968) ; les chercheurs de la Faculté de Médecine de Nantes soupçonnaient, en effet, que ces granulomes étaient causés par des larves d'Ascarides parasitant les Sardines. Notre étude nous avait montré que les Sardines étaient parasitées par des larves appartenant au genre Thynnascaris; il ne nous avait pas été possible, en nous basant uniquement sur les caractères des larves, de faire une détermination spécifique.

Nous pourrions réaliser une détermination spécifique certaine si nous obtenions expérimentalement le développement de ces larves jusqu'au stade adulte dans un Poisson carnassier, mais ceci présente des difficultés matérie!les ; il nous faudrait d'une part faire vivre en captivité pendant assez longtemps des Poissons carnassiers, d'autre part débarrasser ces Poissons au préalable de toute infestation spontanée. C'est pourquoi nous avons cherché à identifier ces larves d'une manière indirecte par une comparaison anatomique et histologique avec les adultes de Thynnascaris rencontrés chez les Poissons carnassiers de la région; nous avons pour ceci récolté les Nématodes d'un certain nombre d'espèces de Poissons pêchés par les pêcheurs du Croisic au cours des mois de juillet et août 1968 et nous en donnons ci-dessous l'étude ; à côté des Ascarides larvaires 
et adultes, nous avons récolté quelques espèces appartenant à d'autres groupes, que nous signalons également dans ce travail.

Les Poissons que nous avons étudiés sont vidés sur les lieux mêmes de pêche, et nous avons effectué nos réco'tes sur les viscères que les pêcheurs nous conservaient dans des sacs; nous avons donc récolté uniquement les parasites qui se trouvaient dans le tube digestif ou sur la paroi de ce tube et dans le foie.

\section{Nematodes appartenant à d'autres groupes que les Ascarides}

Nous avons récolté au cours de notre enquête trois espèces n'appartenant pas aux Ascarides.

\section{A. - Cucullanus hians (Dujardin 1845).}

Nous avons rencontré cette espèce chez Conger vulgaris Cuv.

Nos spécimens correspondent à la description de l'espèce donnée par CampanaRouget et Chabaud (1956).

Cependant, la queue de la femelle est chez nos spécimens déviée vers l'arrière (fig. 1, A), alors qu'elle est dans le prolongement du corps chez les spécimens de Cam. pana-Rouget et Chabaud. Les spicules sont également plus longs chez nos spécimens (1,6 mm chez un mâle de $13 \mathrm{~mm}$ au lieu de $1,26 \mathrm{~mm}$ chez un mâle de $15 \mathrm{~mm}$ ); enfin, on observe chez nos spécimens une papille post-anale supplémentaire (fig. 1, B).
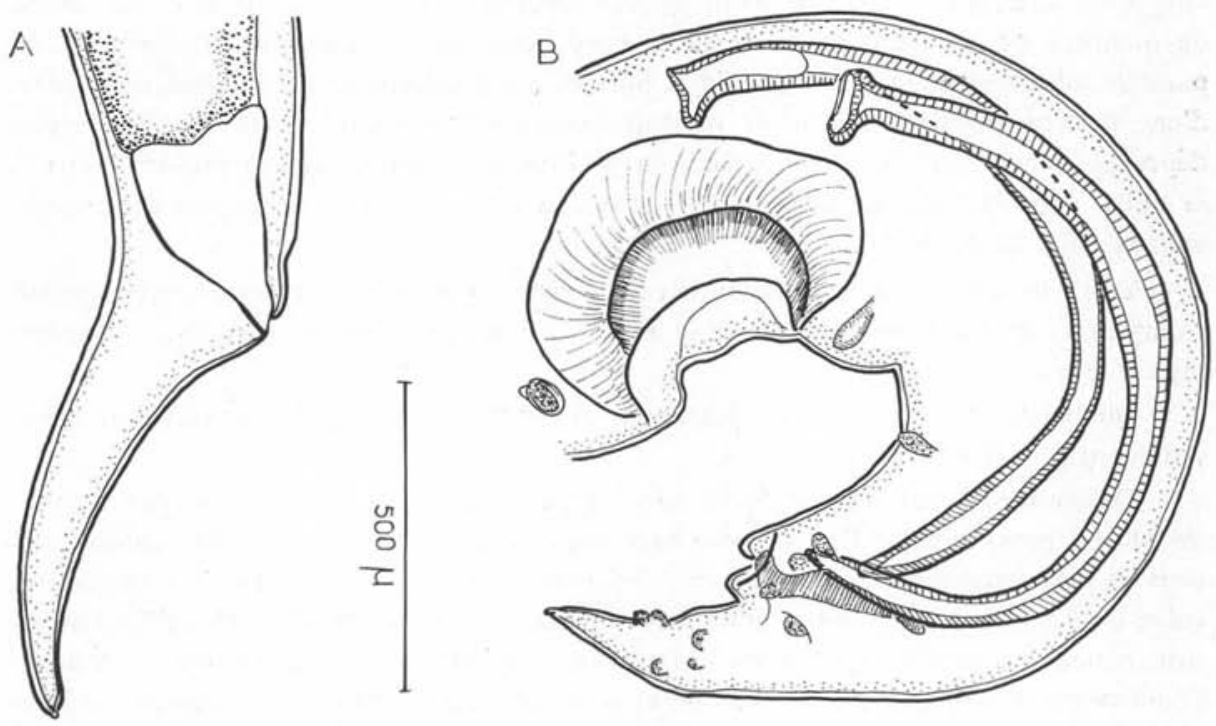

FIg. 1. - Cucullanus hians (Dujardin 1845). - A) Queue de la femslle, vue latérale. B) Queue du mâle, vue latérale 
B. - Proleptus obtusus (Dujardin 1845).

Nous avons rencontré cette espèce en grande abondance chez Scylliorhinus canicula $L$. Nos spécimens correspondent aux descriptions de l'espèce données par Seurat (1919) et Müller (1925).

Elle est aisément identifiable par son spicule muni d'une bosse dorsale située à un peu plus du tiers de sa longueur depuis l'extrémité distale.

\section{C. - Spinitectoides berlandi n.g., n. sp.}

Nous avons trouvé respectivement une larve chez Gadus luscus L. et trois femelles adultes chez Molva molva (L.) d'une espèce de Spiruride déjà décrite uniquement sur des femelles par Berland (1961) chez Molva molva sous le nom de Spinitectus sp.

DESCRIPTION : Nématode long et grêle; dans la région antérieure, chaque anneau cuticulaire porte sur son bord postérieur une vingtaine de festons arrondis; ceux-ci deviennent de moins en moins marqués vers l'arrière, pour disparaître à peu près complètement un peu en avant de la limite œsophage-intestin; pharynx court, évasé en entonnoir dans sa région antérieure; sa paroi est épaisse et creusée d'une gouttière circulaire formant un anneau immédiatement en arrière de la bouche ; œsophage divisé en une portion musculaire courte et une portion glandulaire beaucoup plus longue ; deirides en formes de petites épines; anneau nerveux situé à la limite pharynx-œsophage, pore excréteur légèrement en dessous de l'anneau nerveux (fig. $2 \mathrm{D}$ ).

L'ouverture buccale est losangique et porte sur ses angles latéraux deux petites languettes dressées; immédiatement sous l'ouverture buccale, se trouve un cercle de quatorze petites dents insérées sur le bord postérieur de l'anneau; il existe quatre papilles submédianes et deux amphides. Sur un cercle interne à ces papilles, on observe d'une part en position dorsale et ventrale deux petits boutons situés dans une légère dépression de la surface, d'autre part, en position submédiane quatre prolongations de la pulpe dans la cuticule antérieure; l'ensemble de ces formations simule des papilles internes (fig. 2, A, B, C).

Vulve un peu en arrière du milieu du corps; deux utérus; œufs à coque épaisse, embryonnés au moment de la ponte et munis à l'un des pôles de deux longs filaments (fig. 2, F).

Queue de la femelle droite, portant à son extrémité une paire de phasmides bien visibles (fig. 2, E).

Dimensions d'une femel'e de $53 \mathrm{~mm}$ : largeur maximum : $640 \mu$; pharynx : $100 \mu$; œsophage musculaire : $1.000 \mu$; œsophage glandulaire : $5.000 \mu$; deirides, anneau nerveux et pore excréteur respectivement à $65 \mu, 110 \mu$ et $130 \mu$ de l'extrémité antérieure ; vulve à $29,5 \mathrm{~mm}$ de l'extrémité antérieure ; queue : $225 \mu$; œufs $45 \mu / 35 \mu$; la première strie cuticulaire débute à $100 \mu$ de l'extrémité antérieure, elle ne porte pas de festons ; la deuxième, située à $30 \mu$ de la première, porte des festons très petits ; les stries suivantes portent des festons bien développés et sont espacées les unes des autres de $35 \mu$ à $45 \mu$; chaque feston a une hauteur de $20 \mu$ environ; vers l'arrière, les festons devien- 


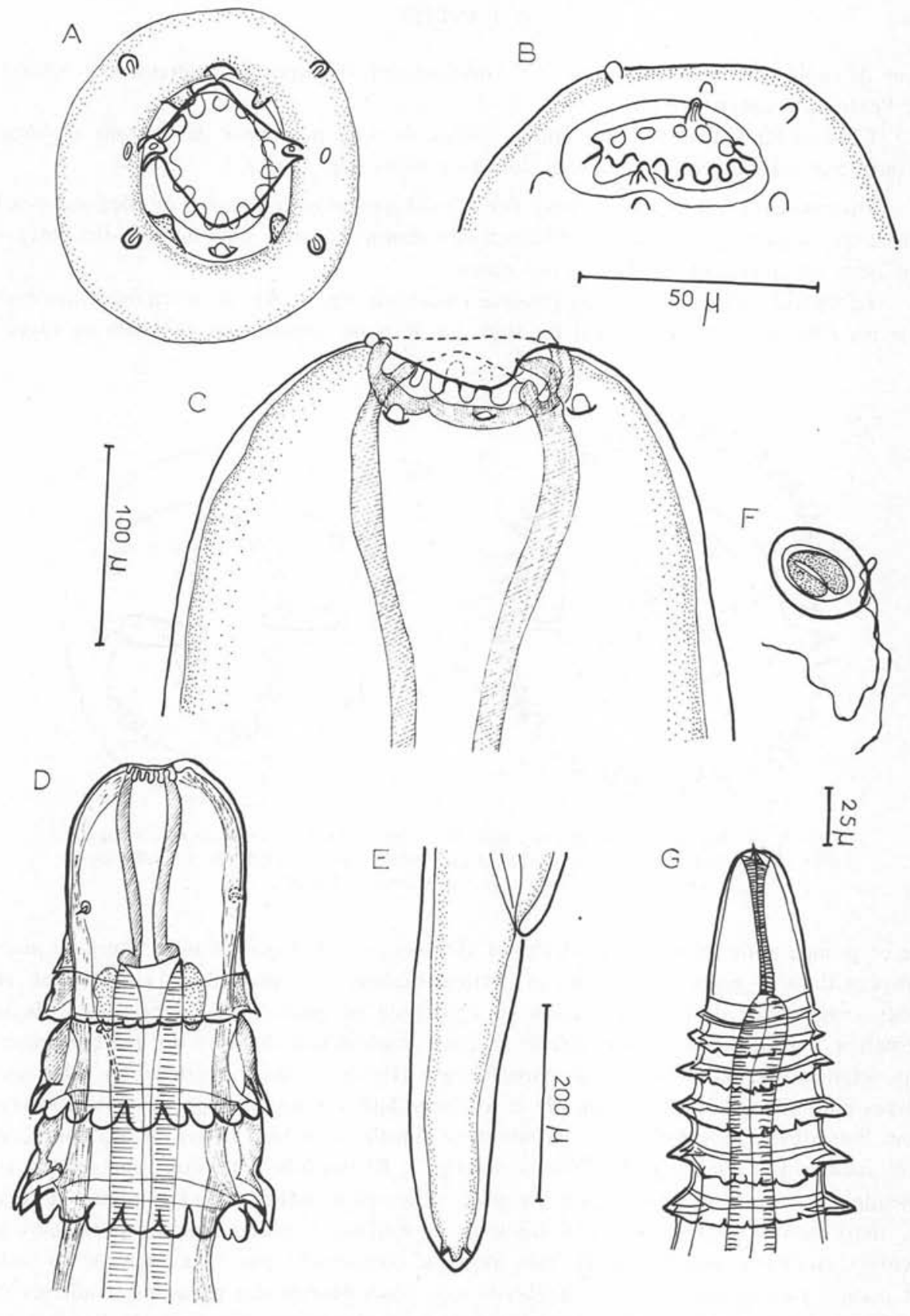

FIG. 2. - Spinitectoides berlandi n. g., n. sp., femelle. A) Extrémité antérieure, vue apicale. B) Extrémité antérieure, vue latérale. C) Extrémité antérieure, vư médiane. D) Région antérieure, vue latéro-médiane. E) Queue. F) CEuf. G) Larve, extrémité antérieure. (A, B, C : échelle $50 \mu ; \mathrm{E}$ : échelle $200 \mu ; \mathrm{D}, \mathrm{G}$ : échelle $100 \mu$; F : échelle $25 \mu$ ) 
nent de moins en moins marqués et l'ornementation disparaît complètement à $5.300 \mu$ de l'extrémité antérieure.

Chez 'a larve trouvée chez Gadus luscus, le bord postérieur des festons est droit et non pas arrondi comme chez les formes adultes (fig. 2, G.).

Discussion : Notre description correspond exactement à celle de Berland, sauf en ce qui concerne les œufs, qu'il décrit sans filaments, mais sans doute a-t-il observé des œufs qui n'étaient pas tout à fait mûrs.

La capsule buccale de coupe presque circulaire (fig. 2, A) est un élément qui évoque les Rhabdochoninae, mais il faudrait que le mâle apporte des éléments en faveur
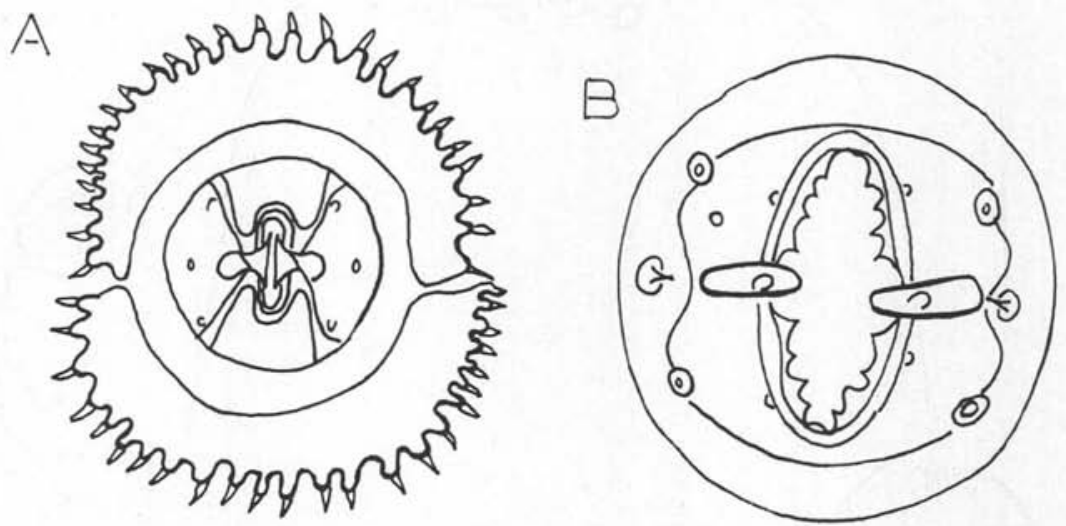

FIG. 3. - A) Spinitectus sp. vue apicale; d'après Dollfus et Campana-Rouget, 1956. B) Cystidicola stigmatura (Jos. Leidy 1886) (dessin inédit de Camille Desportes) ; d'après Dollfus et Campana-Rouget, 1956

de ce groupe pour envisager de véritables affinités avec cette sous-famille. Berland place l'espèce dans le genre Spinitectus, et l'ornementation en festons de la cuticule est, en effet, très proche de l'ornementation en épines de ce genre ; cepenđant l'étude de sa structure apicale nous montre qu'elle diffère complètement de celle du genre Spinitectus, étudiée par Dollfus et Campana-Rouget (1956); celle-ci possède, en effet, des lèvres chitinoïdes dorsale et ventrale et les lobes latéraux représentant les pseudo-lèvres sont bien développés (fig. 3, A) ; la structure apicale de notre espèce, est, au contraire, très voisine de celle du genre Cystidicola (fig. 3, B), les lobes latéraux représentant les pseudo-lèvres sont même dans notre espèce encore plus rudimentaires et réduits à l'état de deux petites languettes. Nous hésitons, cependant, à placer notre espèce dans le genre Cystidicola, puisque, d'une part, nous ne connaissons pas les caractères du mâle et d'autre part, parce que les Cystidicola sont généralement des parasites tissulaires de Poissons d'eau douce; enfin, son ornementation cuticulaire très spéciale l'écarte des autres espèces du genre; nous créons donc, pour elle, un genre nouveau: Spinitectoides n.g. 
DiAGNOSE: Cystidicolinae; ouverture buccale losangique, portant sur ses angles latéraux deux petites languettes; pharynx évasé à sa partie antérieure; dans la région antérieure, les stries cuticulaires transversales sont ornées d'une série de festons. Parasites du tube digestif des Poissons. Espèce type :

Spinitectoides berlandi, n. sp.

\section{Ascarides.}

\section{Larves.}

Nous avons récolté des larves d'Ascarides appartenant à deux types : A. - Larves de Stomachus Goeze in Zeder 1800 (= Anisakis Dujardin 1845).

De très nombreuses larves appartenant à un premier type morphologique ont été trouvées encapsulées sur le foie ou la paroi du tube digestif des Poissons suivants: Pagellus centrodontus Del., Lophius piscatorius L., Godus luscus L., Merluccius vulgaris Flem., Conger vulgaris Cuv.

Ces larves sont relativement grandes $(20$ à $30 \mathrm{~mm})$ et d'aspect robuste ; à l'état frais, elles se reconnaissent immédiatement à la loupe et même à l'œil nu par leur ventricule œsophagien noir.

Leur extrémité antérieure est munie d'une dent ventrale dont la pointe est dirigée ventralement (fig. 4, A); en vue apicale (fig. 4, B) cette dent est en forme de V; la bouche est triangulaire et il existe quatre grosses papilles sub-médianes; chez les larves les plus grandes, on distingue nettement sous la cuticule larvaire l'ébauche des trois lèvres du stade suivant (fig. $4, \mathrm{C}$ ) ; le pore excréteur est situé très antérieurement, à $40 \mu$ de la dent ventrale; l'œsophage se termine par un ventricule œsophagien assez allongé, dont la limite de séparation avec l'intestin est dirigée obliquement (fig. 4, D) ; la queue se termine par un petit mucron (fig. $4, \mathrm{E}$ ) ; à l'inverse des larves du type suivant, où elle est très développée, l'ébauche génitale est ici invisible ; en coupe histologique, on met en évidence la présence d'un organe excréteur percé d'un canal, situé ventralement le long de l'œsophage, du ventricule et de l'intestin antérieur (fig. 5).

Nous donnons les principales dimensions d'une larve longue de $27 \mathrm{~mm}$ : largeur maximum : $420 \mu$; pore excréteur à $40 \mu$ et anneau nerveux à $340, \alpha$ de l'extrémité antérieure; œsophage long de $2550 \mu$; ventricule long de $1050 \mu$, queue longue de $100 \mu$ avec un mucron de $10 \mu$.

Les larves de ce type sont très communes et ont été décrites par de nombreux auteurs [voir à ce sujet Punt (1949), Dollfus (1953), Berland (1961)] sous le nom d'Anisakis sp. La position du pore excréteur sous la dent ventrale, l'existence de la glande excrétrice et du ventricule œsophagien, liée à l'absence d'un appendice œsophagien et d'un caecum intestinal, permettent en effet de les attribuer au genre Stomachus Goeze in Zeder 1800 avec lequel, selon la classification d'Hartwich (1957), Anisakis tombe en synonymie.

Dans l'état actuel de nos connaissances, il ne nous est pas possible de les attribuer à une espèce déterminée. 

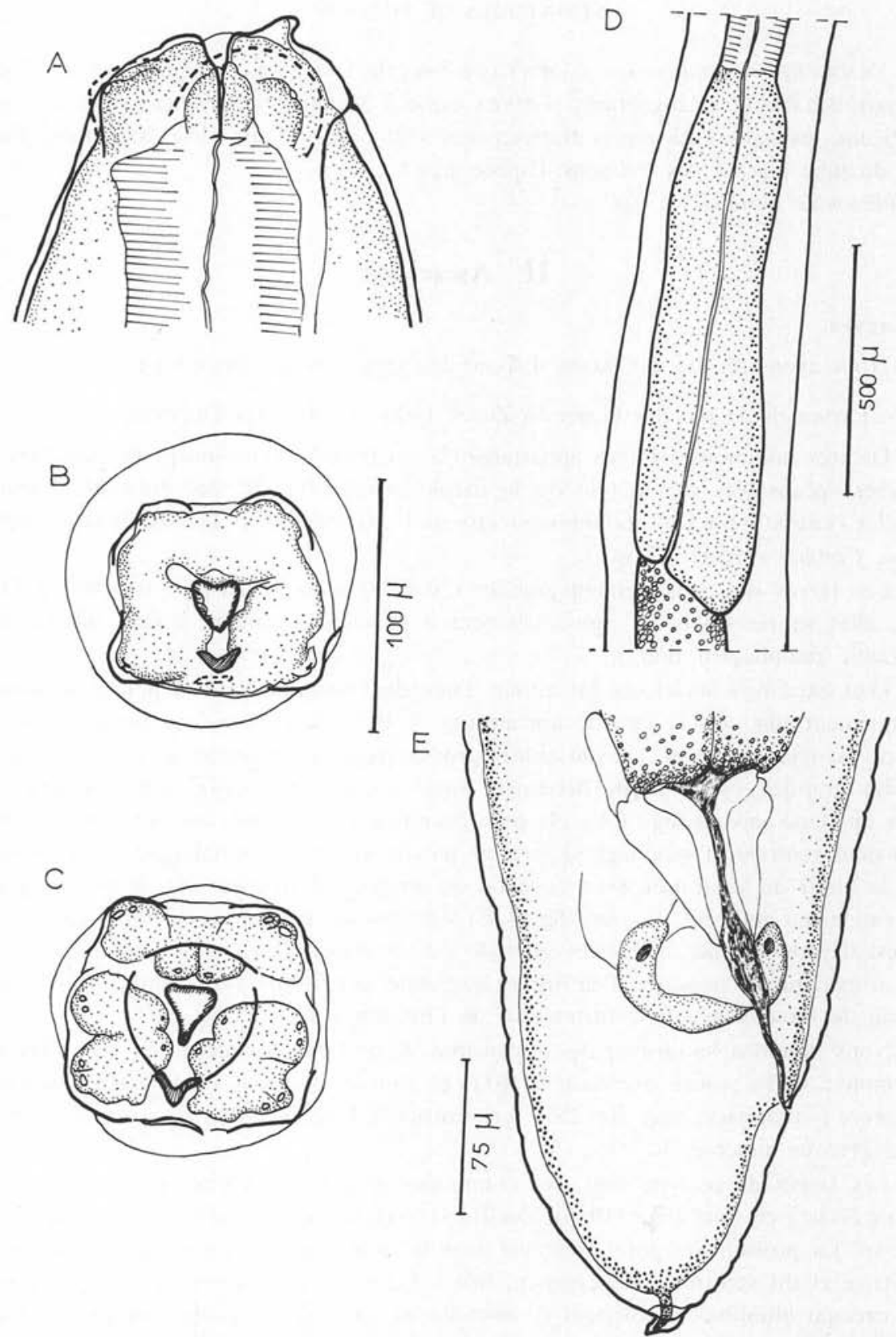

FIG. 4. - Larve de Stomachus sp. (= Anisakis Dujardin). A) Extrémité antérieure, vue latérale. B) Extrémité antérieure, vue apicale. C) Extrémité antérieure d'une larve âgée, vue apicale avec l'ébauche des trois lèvres du stade suivant sous la cuticule larvaire. D) Ventricule csophagien. E) Queue, vue latérale. (A, B, C : échelle $100 \mu$; D : échelle $500 \mu ; \mathrm{E}$ : échelle $75 \mu$ 


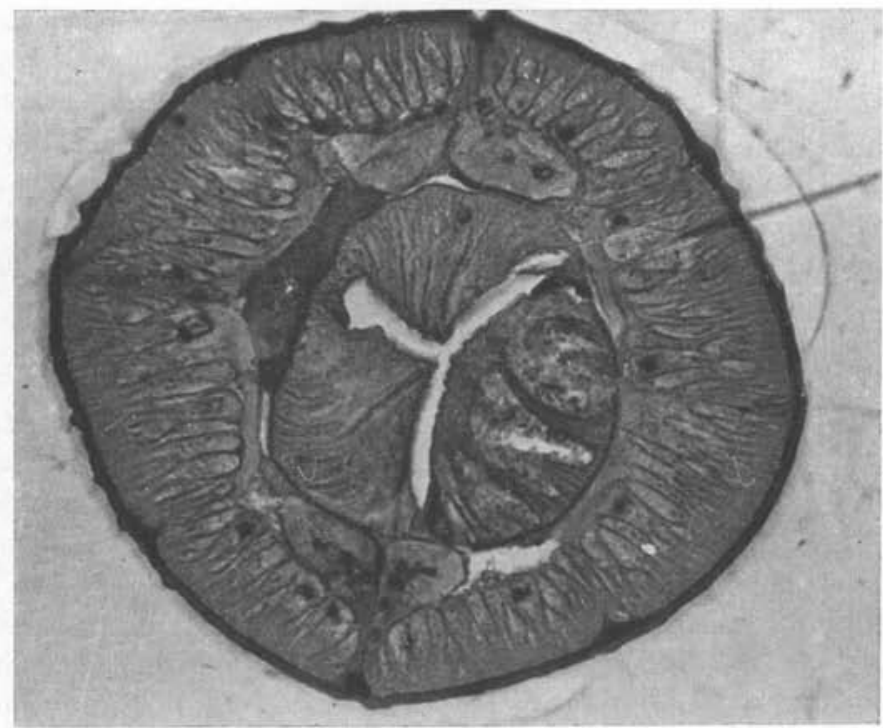

Fig. 5. - Larve de Stomachus sp. (= Anisakis Dujardin), coupe histologique transversale au niveau de l'œsophage, montrant l'organe excréteur (grossissement : 400)

B. - Larves de Tynnascaris aduncum. (Rud. 1802).

Nous avons donné (1969) une description détailiée des larves de ce type récoltées chez les Sardines [Sardina pilchardus (Walbaum)]. Nous avons recueilli des larves du même type morphologique chez les Poissons suivants: Trigla lucerna L., Pagellus centrodontus Del., Lophius piscatorius L., Gadus luscus L., Merluccius vulgaris Flem.

\section{Adultes.}

Nous avons distingué parmi les adultes trois espèces différentes (1):

\section{A. - Thynnascaris aduncum (Rud.).}

L'espèce de beaucoup la plus fréquente est Thynnascaris aduncum; nous l'avons rencontrée chez de nombreux Poissons : Lophius piscatorius L., Gadus luscus L., Merluccius vulgaris Flem., Molva molva (L.), Rhombus maximus L., Solea vulgaris Quensel (2), et Conger vulgaris Cuv.

Elle a été bien décrite par de nombreux auteurs, en particulier par Punt, et nous renvoyons à sa description.

(1) Nous avons groupé avec les adultes les larves du $4^{e}$ stade dont la structure céphalique est identique.

(2) Nous avons trouvé chez Solea vulgaris un seul mâle dont la taille $(68 \mathrm{~mm})$ dépasse de beaucoup celle des mâles recueillis chez les autres Poissons, qui est en moyenne de $30 \mathrm{~mm}$; cependant, la Sole est un poisson de petite taille et devrait donc plutôt, d'après Punt, héberger de petits spécimens; ceci nous a fait supposer que nous avons peut-ếre chez la Sole une sous-espèce différente; nous ne pouvons cependant en décider sur un individu unique et seule une étude ultérieure sur de nombreux spécimens nous permettra de conclure. 
Cette espèce présente de grandes variations de taille; à celles-ci s'ajoutent des variations dans la forme des lèvres et ceci a entraîné plusieurs auteurs (en particulier Schuurmans-Stekhoven, 1935), à distinguer parmi les Thynnascaris parasites de Poissons de mer un certain nombre d'espèces très voisines ; cependant Punt (1941) a montré qu'il s'agissait en réalité d'une seule espèce très répandue et peu spécifique ; aux observations de Punt sur les variations individuelles dans la forme des lèvres, nous ajouterons que chez un même individu la lèvre dorsale peut avoir une forme différente des lèvres latéro-ventrales (fig. $6 \mathrm{~A}, \mathrm{~B}$ ).

\section{DÉVELOPPEMENT DES GEUFS.}

Nous avons recueilli des femelles mûres contenant des œufs chez Lophius piscatorius, Merluccius vulgaris et Rhombus maximus; à chaque fois, nous avons dilacéré les utérus et mis les œufs en culture dans des boîtes de Pétri contenant une couche d'eau de mer d'environ $1 / 2 \mathrm{~cm}$; les boîtes de Pétri étaient placées à la lumière du jour à la température ambiante.

Les œufs provenant des parasites de Lophius piscatorius et Merluccius vulgaris ne s'étaient pas développés au-delà du stade morula au bout d'un mois et la culture a été interrompue. Par contre, les œufs provenant des parasites de Rhombus major ont montré un développement rapide. Après quelques jours, par pression sur la coque d'un œuf, nous avons pu faire sortir une larve qui correspond au premier stade larvaire ; elle est longue de $265 \mu$, ne possède pas de dent cuticulaire apicale ; l'œsophage, peu visible, mesure environ $90 \mu$; la queue, très longue et effilée, mesure $65 \mu$ (fig. 6, D).

Au bout de sept jours, des larves écloses sont apparues dans la culture. Ces larves du deuxième stade ont le même aspect que celles décrites par Punt (fig. 6, C) : elles sont entourées par une enveloppe cuticulaire très lâche dans laquelle elles remuent activement; l'extrémité antérieure est munie d'une petite dent chitineuse ; l'œsophage paraît vide; à sa limite avec l'intestin, on observe deux petits appendices, ébauches du caecum intestinal et de l'appendice œsophagien; l'intestin a dans sa moitié postérieure un contenu granuleux.

Dimensions d'une larve de $310 \mu$ : largeur maximum: $15 \mu$; longueur de l'œsophage : $90 \mu$; longueur de la queue : $50 \mu$; longueur de l'enveloppe cuticulaire : $395 \mu$; largeur maximum : $35 \mu$.

L'enveloppe cuticulaire, qui correspond à la première mue larvaire, possède des stries transversales, débutant à $25 \mu$ de son apex; l'extrémité antérieure montre une petite pièce longue d'une dizaine de $\mu$ dirigée vers l'intérieur ; l'extrémité distale est très longue et effilée.

Après sept jours, on trouve encore dans la culture, à côté des larves écloses, des œufs à tous les stades de développement.

\section{B. - Thynnascaris rigidum (Rud. 1809).}

Nous avons trouvé cette espèce chez Lophius piscatorius, associée avec Thynnascaris aduncum; elle a été bien décrite par Stefanski (1936) sous le nom de Contra- 


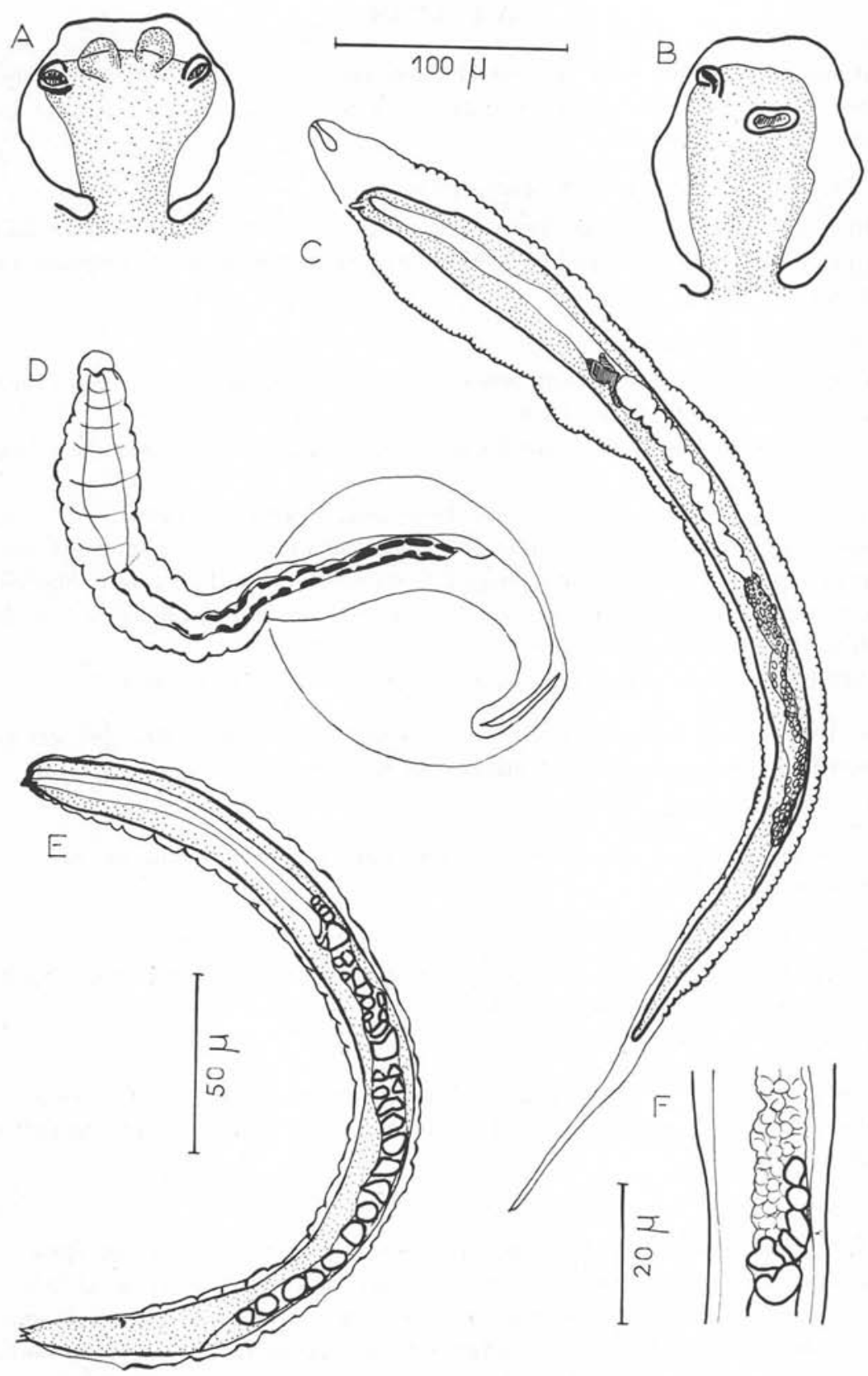

F1G. 6. - A, B, C, D. - Thynnascaris aduncum (Rud.). A) Lèvre dorsale. B) Lèvre latéro-ventrale du même individu. C) Larve à l'éclosion. D) Larve du premier stade obtenue par pression sur la coque de l'œuf. E, F) Thynnascaris cornutum (Stóssich), larve à l'éclosion. E) Larve entière. F) Limite œsophago-intestinale. A, B : échelle $100 \mu ; C, D, E$ : échelle $50 \mu$; E : échelle $20 \mu$ 
caecum lophii, puis par Punt qui rétablit son nom original de rigidum donné par Rudolphi et attribué à tort à des spécimens de Thynnascaris aduncum par les auteurs suivants.

C. - Thynnascaris cornutum (Stossich, 1904).

Nous avons rencontré cette espèce chez Thynnus germo Lacép. Elle a été bien décrite par Baylis (1923), par Dollfus (1933, 1935) sous le nom de Thynnascaris Legendrei et par Berland (1961).

\section{DÉVELOPPEMENT DES CEUFS :}

Nous avons mis les œufs d'une femelle mûre en culture dans les mêmes conditions que ceux de Thynnascaris aduncum.

Au bout de sept jours, de nombreuses larves sont écloses et s'agitent au fond du cristallisoir.

Ces larves (fig. 6, E) sont entourées d'une cuticule de mue formant un manchon beaucoup moins lâche que chez celles de Thynnascaris aduncum; elles possèdent une petite dent cuticulaire apicale ; l'œsophage est peu visible ; à sa limite avec l'intestin, on observe l'ébauche d'un caecum intestinal et d'un appendice œsophagien (fig. 6, F) ; l'extrémité caudale a la forme d'une fourche à trois dents.

Chez une larve de $300 \mu$, l'œsophage mesure $100 \mu$ et la queue $5 \rho_{\mu}$

Les trois espèces citées ci-dessus se distinguent les unes des autres par des caractères bien nets que nous rappelons brièvement ici :

a) la forme du corps:

Cylindrique chez $T$. aduncum et $T$. rigidum, il est en forme de fuseau chez T. cornutum.

b) l'aspect de la base des lèvres:

Simple chez $T$. aduncum et $T$. cornutum, elle est creusée de rainures formant un dessin complexe chez $T$. rigidum (fig. 7).

c) la place de la vulve :

Celle-ci est située à peu près au $1 / 4$ antérieur du corps chez $T$. cornutum, un peu en arrière du $1 / 3$ antérieur chez $T$. aduncum, et un peu en arrière du milieu du corps chez $T$. rigidum.

d) les épines de la queue (fig. 8).

L'extrémité caudale est dans les deux sexes couverte de grosses épines chez $T$. aduncum; ces épines sont beaucoup plus petites chez $T$. rigidum et elles sont absentes chez $T$. cornutum; cependant, ce caractère n'a pas une valeur absolue, car les épines sont peu visibles et comme effacées chez certains spécimens de $T$. aduncum.

e) la queue de la femelle:

Courte et trapue (moins de $500 \mu$ ) chez $T$. aduncum et $T$. rigidum, elle est longue (plus de $800 \mu$ ) et s'amincit graduellement jusqu'à l'extrémité chez $T$. cornutum (fig. 8). 

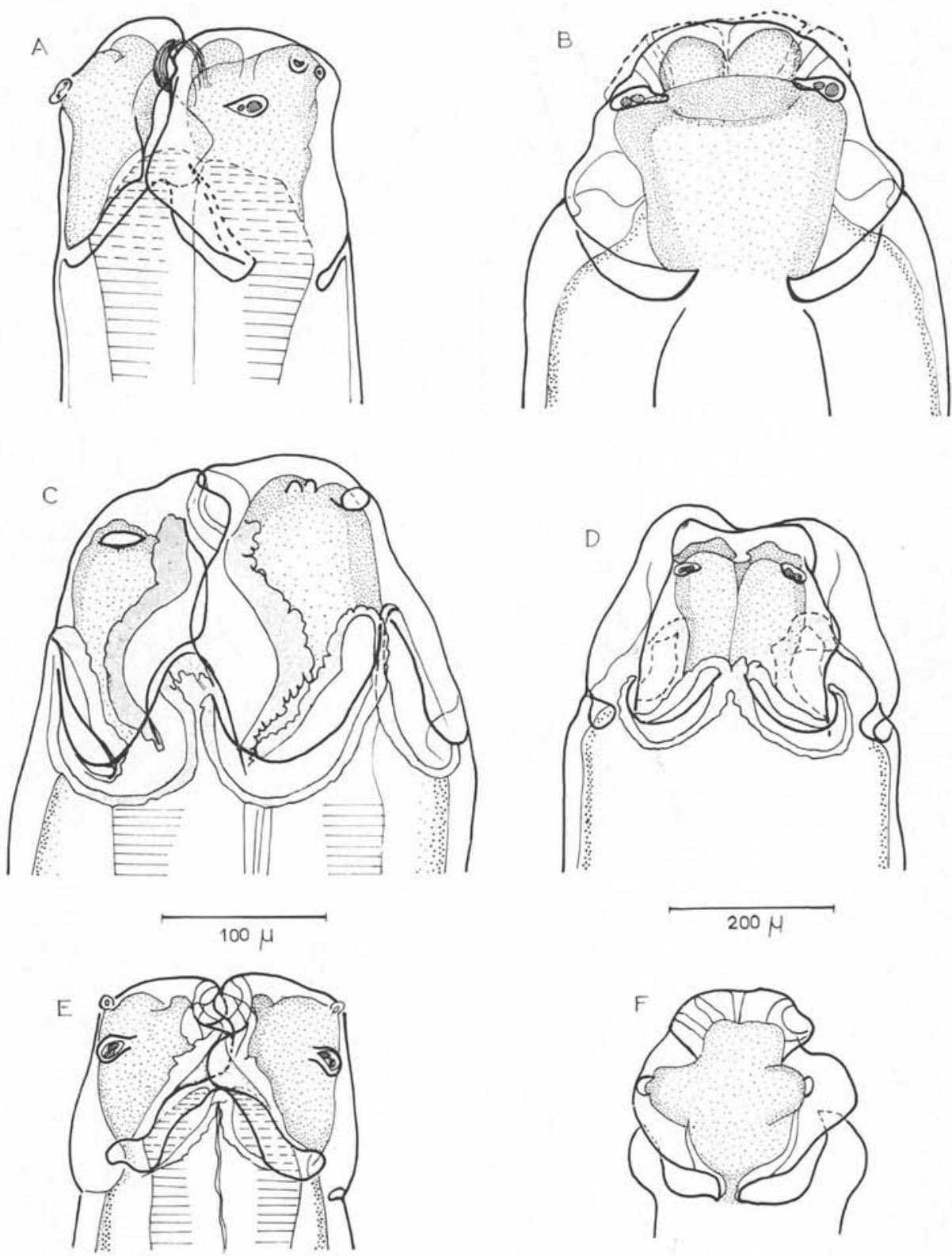

Fig. 7. - Lèvres et Interlabia. - A, B : Thynnascaris aduncum. A) Interlabia. B) Lèvre. C, D) Thynnascaris rigidum. C) Interlabia. D) Lèvre. E, F) Thynnascaris cornutum. E) Interlabia. F) Lèvre. A, B, C, D, E, F, échelle $100 \mu$; D, échelle : $200 \mu$ 

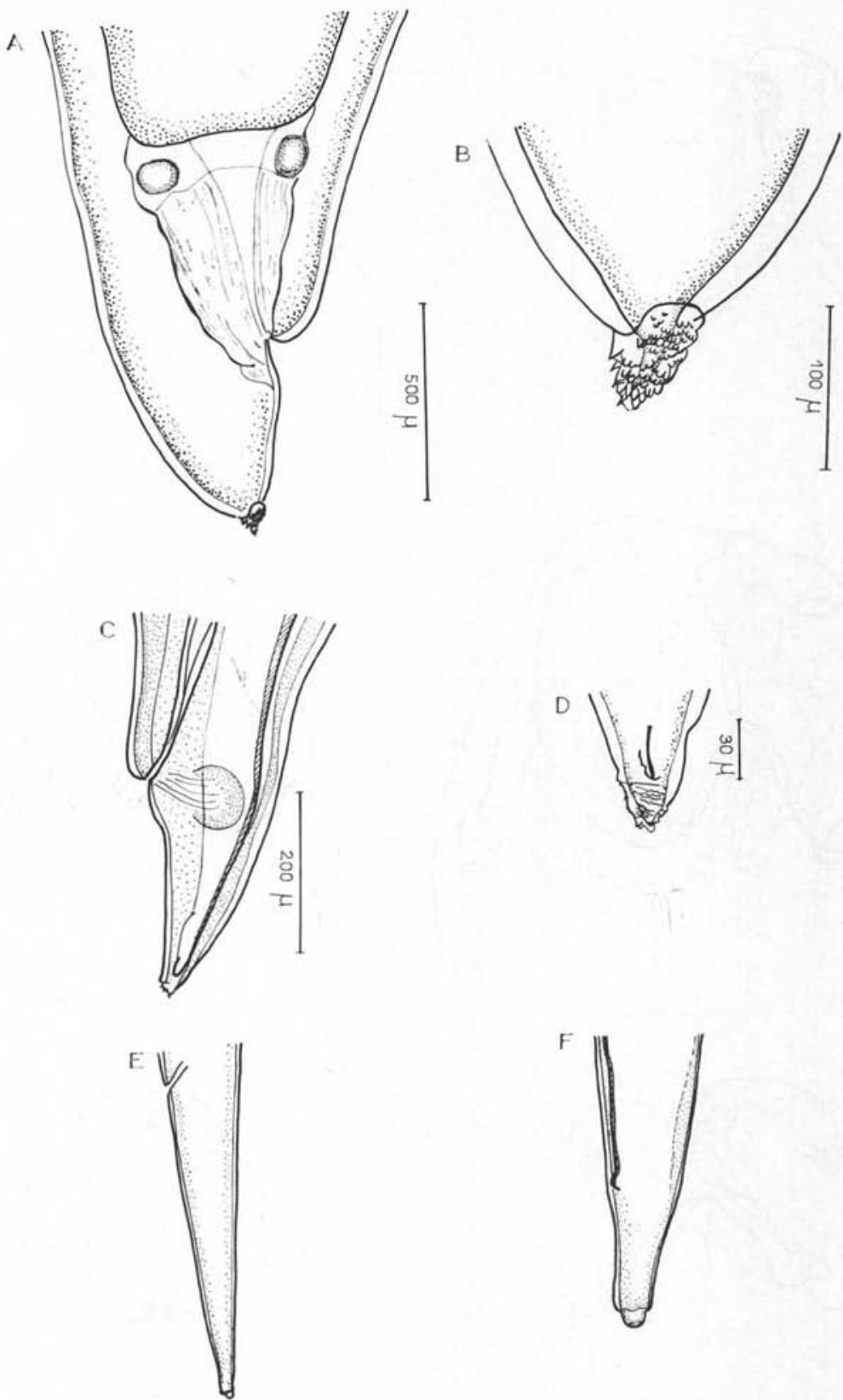

Fig. 8. - Queues des femelles. A, B) Thynnascaris aduncum. C, D) Thynnascaris rigidum. E, F) Thynnascaris cornutum. A, E: échelle $500 \mu$; B, F : échelle $100 \mu ; \mathrm{C}$ : échelle $200 \mu ; \mathrm{D}$ : échelle $30 \mu$ 


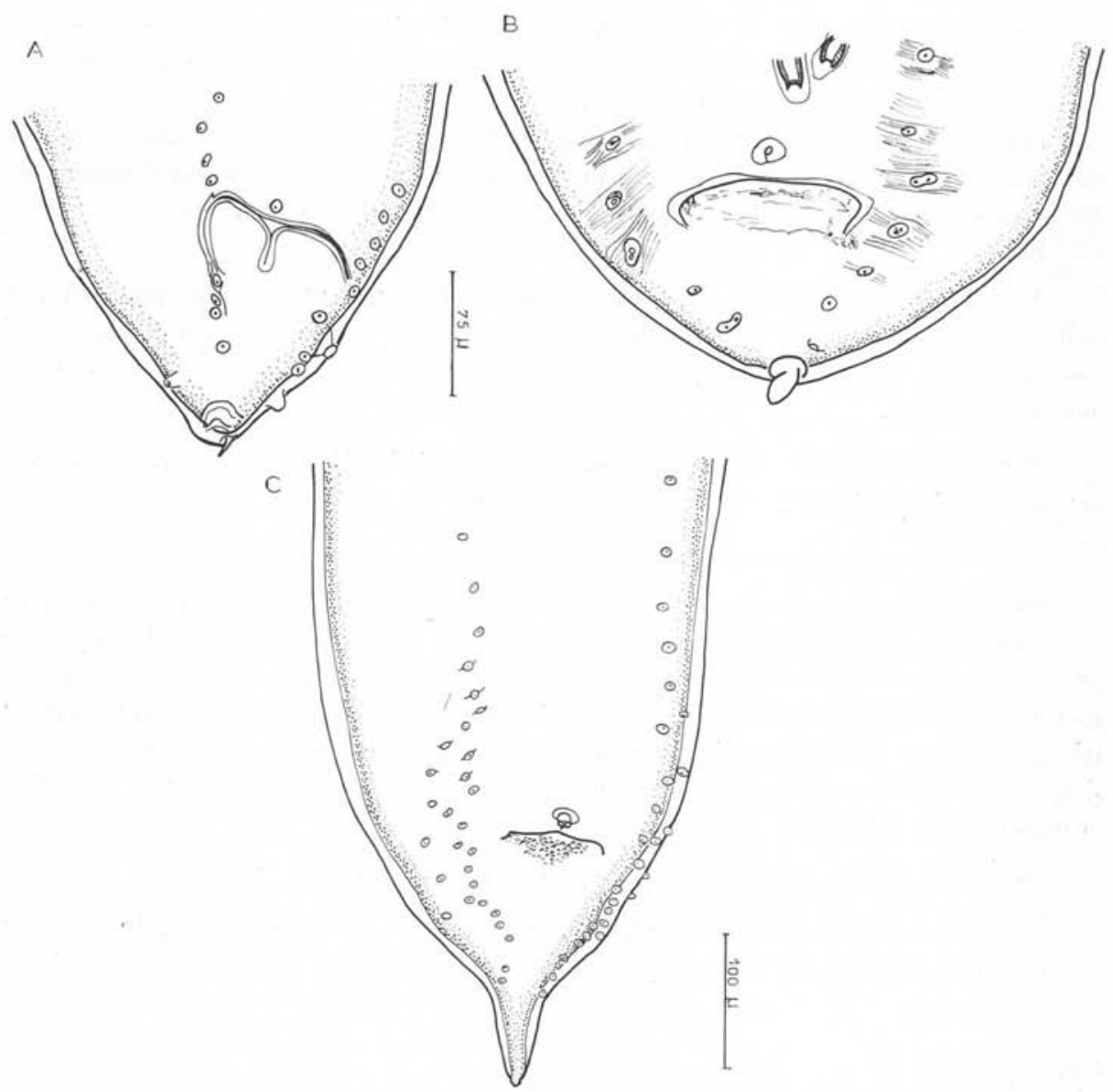

Fig. 9. - Queues des mâles, vues ventrales. A) Thynnascaris aduncum. B) Thynnascaris rigidum. C) Thynnascaris cornutum. A, B) échelle $75 \mu$; C : échelle $100 \mu$

f) la queue du mâle:

Celle-ci a un aspect différent chez chacune des trois espèces : celles de $T$. aduncum et $T$. rigidum sont assez voisines, cependant chez $T$. aduncum, toutes les papilles sont simples alors que chez $T$. rigidum, il en existe des doubles, en particulier celles qui sont au voisinage de l'anus; chez $T$. cornutum, les papilles post-cloacales sont beaucoup plus nombreuses (13 paires) et très petites (fig. 9).

Les spicules sont légèrement plus courts $(900 \mu)$ chez $T$. rigidum que chez les deux autres espèces $(1500$ à $2000 \mu)$. 
g) les longueurs de l'appendice asophagien et du caecum intestinal (fig. 10).

Ceux-ci sont de tailles à peu près égales chez $T$. aduncum où ils mesurent environ le $1 / 3$ de l'œsophage (chez un mâle de $31 \mathrm{~mm}$, œsophage : $3.500 \mu$; appendice œsophagien : $1.100 \mu$ : caecum intestinal : $1.100 \mu$ ).

Chez $T$. rigidum, l'appendice œsophagien est beaucoup plus grand que le caecum intestinal, et sa longueur dépasse largement celle de l'œsophage (chez une femelle de $26 \mathrm{~mm}$, œsophage : $3.000 \mu$; appendice œsophagien : $5.600 \mu$; caecum intestinal : $1.750 \mu)$.

T. cornutum se distingue des deux autres espèces par un caecum intestinal très réduit ; l'appendice œsophagien mesure un peu moins de la moitié de l'œsophage (chez une femelle de $29 \mathrm{~mm}$, œsophage : $3.725 \mu$; appendice œsophagien : $1.550 \mu$; caecum intestinal : $450 \mu)$.

Nous ajoutons à ces caractères deux caractères distinctifs que nous avons observés sur coupes histologiques :

a) La forme de l'appendice asophagien en section transversale.

Des sections transversales des trois espèces montrent que l'appendice œsophagien est constitué sur la plus grande partie de sa longueur chez $T$. aduncum et $T$. cornutum de deux cordons accolés, dont l'un a un plus grand diamètre que l'autre; ces cordons sont p.us gros chez $T$. aduncum que chez $T$. cornutum, où l'appendice osophagien est très grêle ; chez $T$. rigidum, l'appendice œsophagien est constitué par un seul cordon, simplement divisé par un septum médian; d'abord grêle, il s'élargit dans sa moitié postérieure (fig. 11).

b) La forme des ailes latérales en section transversale.

Les ailes latérales sont soutenues intérieurement par une armature chitinoïde dont la forme en section transversale est différente suivant l'espèce (fig. 12).

\section{Conclusion}

Nous avons donc trouvé trois espèces différentes de Thynnascaris adultes chez les Poissons péchés au Croisic (3) et ces espèces sont également celles qui sont le plus fréquemment citées dans la littérature chez les Poissons carnassiers qui fréquentent les mêmes eaux que les Sardines (voir les travaux de Punt et Berland sur les Poissons de la mer du Nord et celui de Stefanski sur ceux du Sud du golfe de Gascogne); d'après Furnestin (1943), les Sardines de la race atlantique septentrionale effectuent, en effet, au cours de leur vie une migration du sud au nord, leur secteur géographique s'étendant de la côte cantabrique jusqu'à la mer du Nord.

(3) Berland signale dans la mer du Nord une $4^{e}$ espèce de Thynnascaris, Thynnascaris incurvum (Rud. 1819) parasite de Xiphias gladius. Cette espèce paraît moins fréquente que les autres dont elle se distingue immédiatement par la grande longueur de cæcum intestinal; la grande taille de ce cæcum la différencie également des larves trouvées dans les Sardines. 

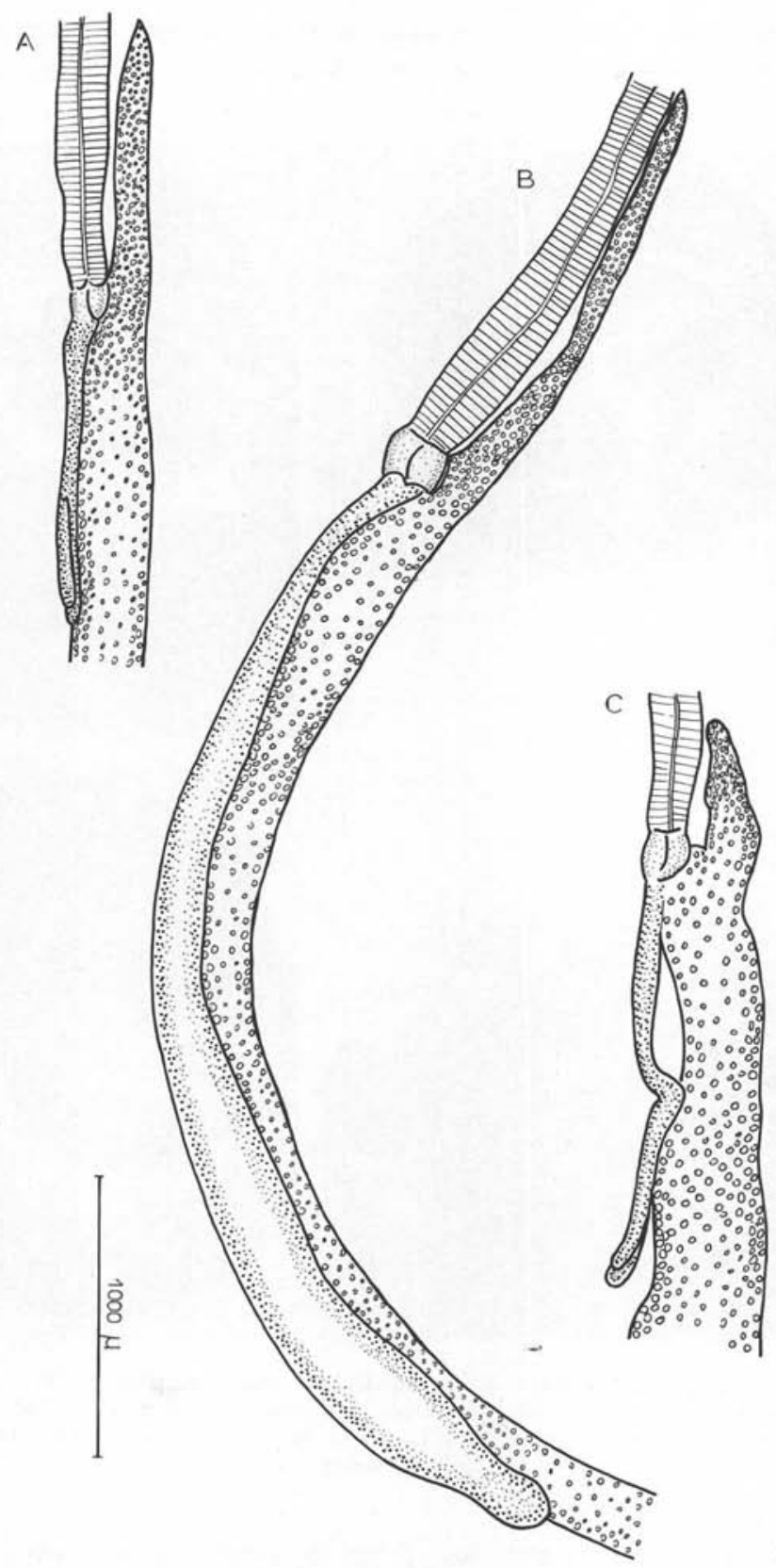

Fig. 10. - Appendices œsophagiens et cæcums intestinaux. A) Thynnascaris aduncum. B) Thynnascaris rigidum. C) Thynnascaris cornutum 
Les longueurs de l'appendice œsophagien et du caecum intestinal, ainsi que la forme de l'appendice œsophagien en section transversale sont semblables chez les larves

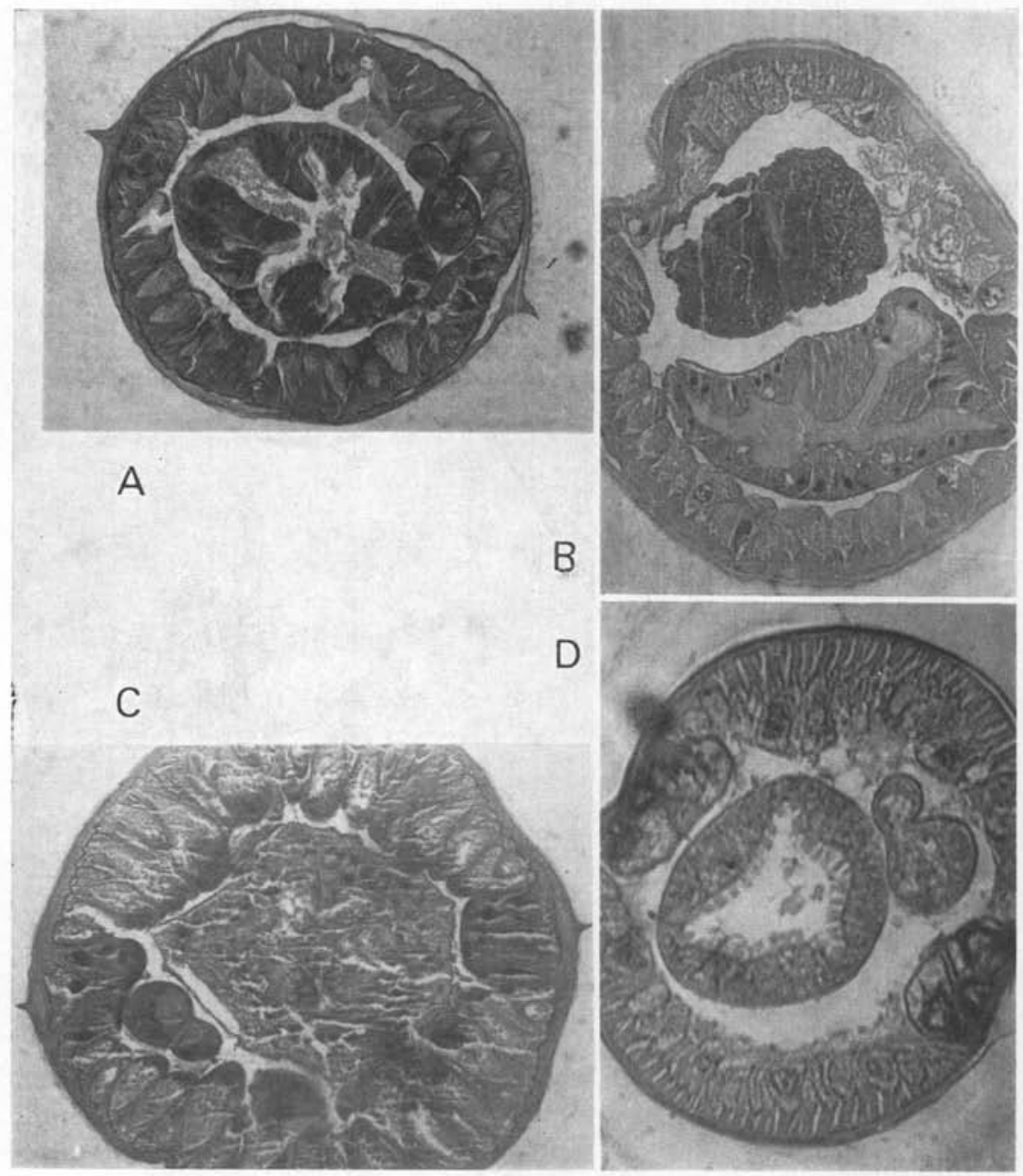

Fig. 11. - Sections transversales au niveau de l'appendice æsophagien. A) Thynnascaris aduncum (grossissement : 75). B) Thynnascaris rigidum (grossissement: 200). C) Thynnascaris cornutum (grossissement: 200). D) Larve de Thynnascaris récoltée dans les Sardines (grossissement : 400)

récoltées chéz les Sardines et chez les adultes de l'espèce Thynnascaris aduncum; de plus les jeunes quatrièmes stades que nous avons obtenus expérimentalement à partir de ces larves chez un Mugil cephalus (Petter, 1969) présentent une extrémité anté- 


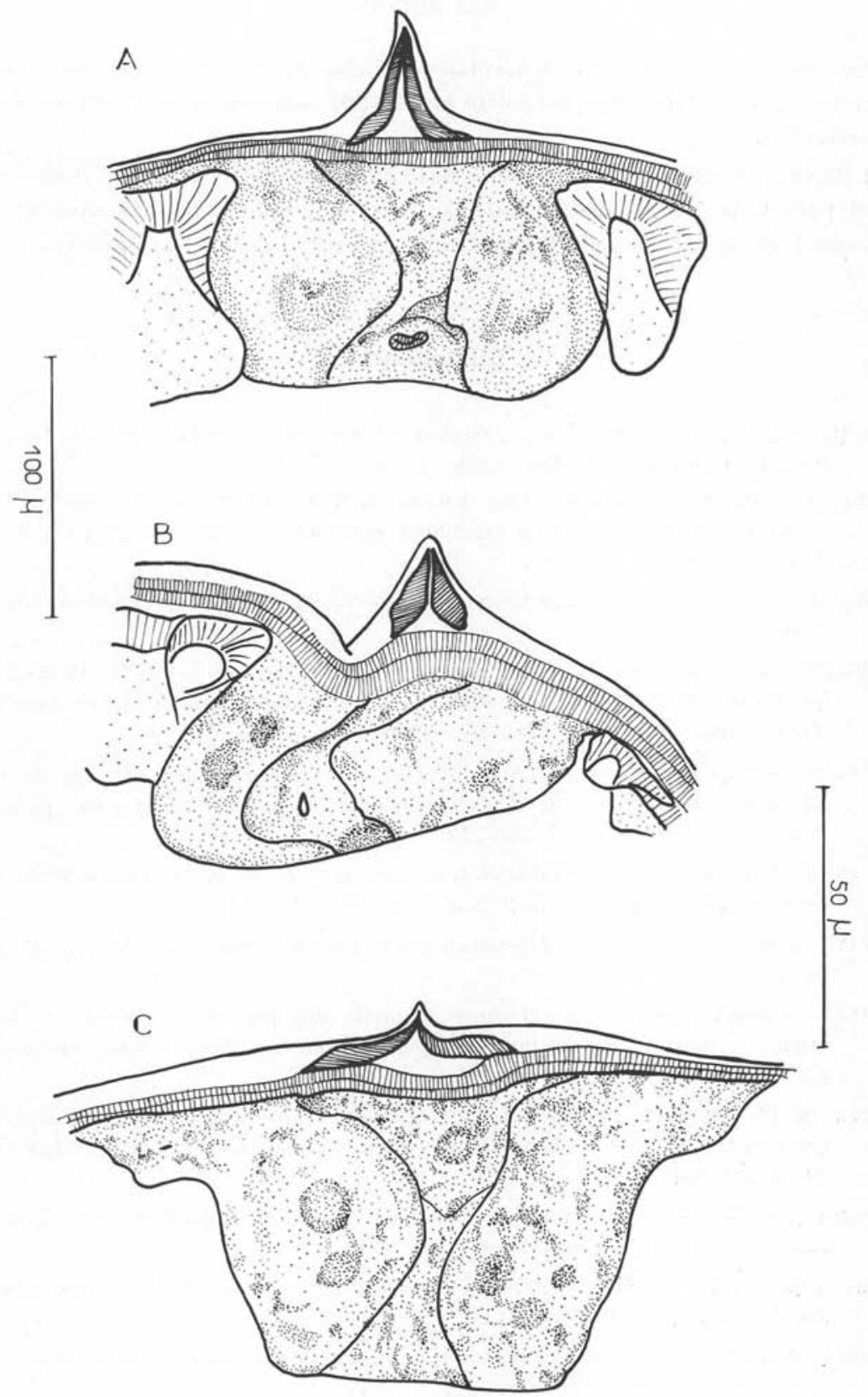

Fig. 12. - Sections transversales des ailes latérales. A) Thynnascaris aduncum. B) Thynnascaris rigidum. C) Thynnascaris cornutum. A) échelle $100 \mu$; B, C : échelle $50 \mu$ 
rieure et une extrémité postérieure identiques à celles de cette espèce ; il est donc à peu près certain que les larves qui parasitent les Sardines appartiennent à l'espèce Thynnascaris aduncum.

L'exposé précédent montre l'intérêt des coupes histologiques dans l'étude des espèces, en particulier dans l'identification des larves, certains caractẻres comme l'aspect en section transversale de l'appendice œsophagien ayant une valeur spécifique.

\section{Bibliographie}

BAYLIS (H. A.), 1923. - Report on a collection of parasitic nematodes, mainly from Egypt. Part. I. Ascaridae and Heterakidae. Parasit., 15, 1-13.

Benatre (A.), Guntz (M.), Simard (Ch.), Lieffring (J.-J.), Bertrand (J.), Forest (J.-L.) et Pelletier (G.), 1968. - Le granulome éosinophilique du tube digestif. Rev. Med. Tours, 2, 237-243.

BerLand (B.), 1961. - Nematodes from some Norvegian marine fishes. Sarsia. Bergen, 2, $1-50$.

Boquien (Y.), Kerneis (J.-P.), Malvy (P.), Kropff (G.), Lenne (H.), Delumeau (G.), Le BoDIC (M.-F.) et LE Bodic (L.), 1966. - Le granulome éosinophile du tube digestif. Arch. Franç. Mal. App. Dig., 55, 977-998.

Campana-Rouget (Y.) et Chabiud (A.-G.), 1956. - Helminthes des environs de Banyuls. III. Sur trois espèces de Cucuilanus (Camallanoidea, Nématoda), parasites de Poissons. Vie et Milieu, 7, 267-279.

Dollfus (R.-Ph.), 1933. - Thynnascaris Legendrei, n. gen., n. sp. de l'estomac du germon, Germo alalonga (Gmel.). Bull. Soc. Zool. Fr., 58, 7-13.

-, 1935. - Sur Contracaecum, Thynnascaris et Amphicaecum. Bull. Soc. Zool. Fr., 60, 88-92.

-, 1953. - Aperçu général sur l'histoire naturelle des parasites animaux de la Morue atlanto-arctique Gadus callarias L. (= Morhua L.). Encyclopedie biologique, 43, 423 pp., Paris.

Dollfus (R.-Ph.) et Campana-Rouget (Y.), 1956. - Une nouvelle espèce d'Ascarophis (Nematoda, Spirurinae) chez Gadus luscus L. Révision du genre. Ann. Parasit., $31,4,385-404$.

Furnestin (J.), 1943. - Contribution à l'étude biologique de la Sardine. Rev. Trav. Office Scient. Techn. Pêches maritimes, 13, $\mathrm{n}^{\circ}$ 1-4.

HARTwich (G.), 1957. - Zur Systematick der Nematoden Superfamilie Ascaridoidea. Zool. Jahrb.. Abt. Syst., 85 (3), 211-252.

Müller (J.-F.), 1925. - Some new features of nematode morphology in Proleptus obtusus Dujardin. J. Parasit., 12, 84-90.

Petrer (A.-J.), 1969. - Enquête sur les Nématodes des Sardines pêchées dans la région nantaise ; rapport possible avec les granulomes éosinophiles observés chez l'Homme dans la région. Ann. Parasit., 44, 25-36. 
Picard (R.), Hardy (M.) et Kerneis (J.-P.), 1954. - Les granulomes éosinophiliques du tube digestif. Arch. Mal. App. Dig., 43, 920-937.

Punt (A.), 1941. - Recherches sur quelques Nématodes parasites de poissons de la Mer du No:d. Mémoires du Musée Royal d'Histoire naturelle de Belgique, 98, 1-110.

SchuURmans-Stekhoven (J.-H.), 1935. - Nematoda parasitica. Grimpe et Wagler, Tierwelt der Nord-und Ostsee, Vc.

Seurat (L.-G.), 1919. - Sur la morphologie du Proleptus obtusus Duj. (Acuariidae). Bull. mus. hist. nat., 3, 166-172.

Stefanski (W.), 1936. - Sur les Nématodes parasites des vives «Trachinus draco» et des baudroies «Lophius piscatorius », Bull. Station biologique d'Arcachon, 33, 59-88. 